\title{
Auditory Electrophysiological Findings in Musicians and Non-Musicians
}

\author{
Mohd Normani Zakaria \\ Audiology and Speech Pathology Programme, School of Health Sciences, Universiti Sains Malaysia, Kelantan, Malaysia
}

Dear Editor,

I read with great interest a recently published article titled "Effect of Music Training on Auditory Brainstem and Middle Latency Responses" considerately written by Choi \& Cho (2020). In essence, ongoing research efforts in this subspecialty area of audiology are indeed warranted and relevant. After gathering the essential data among the college students, the authors concluded that the musical training had significant effects on the brainstem region (as revealed by auditory brainstem response findings), rather than the subcortical area (based on auditory middle latency response results). As such, I would like to highlight several issues that are worthy of consideration to ascertain the respective study outcomes.

In the aforementioned study, auditory brainstem response (ABR) waveforms were recorded using $0.1 \mathrm{~ms}$ click stimuli presented at an intensity level of $80 \mathrm{dBnHL}$ with a stimulus repetition rate of 33/s (Choi \& Cho, 2020). The reported mean wave $\mathrm{V}$ latency was $5.11 \mathrm{~ms}$ for musicians and $5.37 \mathrm{~ms}$ for non-musicians. These mean values were notably shorter than those of previous studies employing comparable ABR test parameters (i.e., around $5.5 \mathrm{~ms}$ ) (Hall, 2007; Samelli et al., 2012; Zakaria et al., 2019). As reported by Samelli et al. (2012), the mean wave V latency was $5.76 \mathrm{~ms}$ for normal adults and $5.68 \mathrm{~ms}$ for musicians without hearing loss. It is however possible to have such shorter ABR latencies if the study participants are mostly females. Shorter ABR latencies in females than in males have been consistently

Received: March 20, 2020 Accepted: December 16, 2020

Correspondence:

Mohd Normani Zakaria, PhD

Audiology and Speech Pathology Programme, School of Health Sciences, Universiti Sains Malaysia, 16150 Kubang Kerian, Kelantan, Malaysia

Tel: +60-9-7677691, Fax: +60-9-7677515

E-mail: mdnorman@usm.my

(c) This is an Open Access article distributed under the terms of the Creative Commons Attribution Non-Commercial License (https://creativecommons.org/licenses/by-nc/4.0) which permits unrestricted non-commercial use, distribution, and reproduction in any medium, provided the original work is properly cited. reported in the literature (Hall, 2007; Jalaei et al., 2017; Jerger \& Hall, 1980; Zakaria et al., 2019). In the study by Choi \& Cho (2020), the gender distribution of participants in both groups was not stated. This information is imperative to support the study findings and conclusions. In fact, significant differences in ABR latencies between the two groups could also be obtained if the number of female participants is not comparable across the groups. If this is the case, performing analysis of covariance (with gender as the covariate), rather than the conventional independent $t$-test is advantageous to produce more realistic and well-controlled study outcomes (Zakaria et al., 2015).

\section{Ethical Statement \\ N/A}

\section{Acknowledgments}

N/A

\section{Declaration of Conflicting Interests}

There are no conflict of interests.

\section{Funding}

N/A

\section{ORCID iD}

Mohd Normani Zakaria https://orcid.org/0000-0002-3694-3460

\section{REFERENCES}

Choi, C. H. \& Cho, H. S. (2020). Effect of music training on auditory brainstem and middle latency responses. Audiology and Speech Research, 16(1), 41-47.

Hall, J. W. (2007). New Handbook of Auditory Evoked Responses. Boston, MA: Pearson.

Jalaei, B., Zakaria, M. N., Mohd Azmi, M. H., Nik Othman, N. A., \& Sidek, D. (2017). Gender disparities in speech-evoked auditory brainstem response in healthy adults: Any relation to head size? The Annals of Otology, Rhinology, and Laryngology, 126(4), 290-295.

Jerger, J. \& Hall, J. (1980). Effects of age and sex on auditory brainstem response. Archives of Otolaryngology, 106(7), 387-391.

Samelli, A. G., Matas, C. G., Carvallo, R. M., Gomes, R. F., de Beija, C. S., Magliaro, F. C., et al. (2012). Audiological and electrophysiological assessment of professional pop/rock musicians. Noise and Health, 14(56), 
6-12.

Zakaria, M. N., Abdul Wahab, N. A., Maamor, N., Jalaei, B., \& Dzulkarnain, A. A. A. (2019). Auditory brainstem response (ABR) findings in males and females with comparable head sizes at supra-threshold and threshold levels. Neurology, Psychiatry and Brain Research, 32, 4-7. Zakaria, M. N., Nik Othman, N. A., \& Cheu Lih, A. (2015). Tinnitus severity and the sound therapy outcome. Clinical and Experimental Otorhinolaryngology, 8(2), 179. 